\title{
ON THE HOMOLOGY OF POSTNIKOV FIBRES
}

\author{
Y. FÉLIX AND J. C. THOMAS
}

(Communicated by Frederick R. Cohen)

\begin{abstract}
Let $k$ be a field of positive characteristic and $X$ be a simply connected space of the homotopy type of a finite type CW complex. The Postnikov fibre $X_{[n]}$ of $X$ is defined as the homotopy fibre of the $n$-equivalence $f_{n}: X \rightarrow X_{n}$ coming from the Postnikov tower $\left\{X_{n}\right\}$ of $X$. We prove that if the Lusternik-Schnirelmann category of $X$ is finite, then $H_{*}\left(X_{[n]} ; k\right)$ contains a free module on a subalgebra $K$ of $H_{*}\left(\Omega X_{n} ; k\right)$ such that $H_{*}\left(\Omega X_{n} ; k\right)$ is a finite-dimensional free $K$-module.
\end{abstract}

Let $k$ be a field of positive characteristic $p$ and $X$ be a simply connected space which has the homotopy type of a finite type CW complex. The Postnikov tower of $X$ consists of a sequence of principal fibrations

$$
X_{n} \stackrel{p_{n}}{\rightarrow} X_{n-1} \rightarrow K\left(\pi_{n}(X), n+1\right)
$$

and of $n$-equivalences $f_{n}: X \rightarrow X_{n}$ satisfying $p_{n} f_{n}=f_{n-1}$. The homotopy fibre of $f_{n}$ is then denoted by $X_{[n]}$ and is called the $n$th Postnikov fibre of $X$,

$$
X_{[n]} \rightarrow X \stackrel{f_{n}}{\rightarrow} X_{n} .
$$

The homotopy lifting property of the fibration $f_{n}$ defines a natural action of $H_{*}\left(\Omega X_{n} ; k\right)$ on $H_{*}\left(X_{[n]} ; k\right)$ [8]. This action is called the holonomy operation and its behaviour in this context is the subject of this paper.

More generally we will consider a fibration of simply connected spaces

$$
F \rightarrow E \stackrel{f}{\rightarrow} B
$$

such that $\Omega B$ has a stable $r$-stage Postnikov system [7]. This means that $\Omega B$ can be obtained by a finite sequence of multiplicative fibrations

$$
G_{r} \rightarrow G_{r-1} \rightarrow K_{r}, \quad r=0, \ldots, n, \quad \Omega B=G_{n}, \quad G_{-1}=\{*\},
$$

with $K_{r}$ a product of Eilenberg-Mac Lane spaces. The spaces $\Omega X_{n}$ are stable $n$-stable Postnikov systems. This happens also, for instance, when $B$ has only a finite number of nonzero homotopy groups. We can now state our main theorems.

Received by the editors April 25, 1991.

1991 Mathematics Subject Classification. Primary 55S45, 57T05.

Key words and phrases. Eilenberg-Mac Lane spaces, Postnikov tower, grade of a module, Lusternik-Schnirelmann category of a space. 
The category of $f: E \rightarrow B$, cat $f$, is defined as the least $m \leq \infty$ such that $E$ can be covered by $m+1$ open sets $U_{i}$ so that the restriction of $f$ to each $U_{i}$ is homotopic to zero [1]. Clearly cat $f \leq \operatorname{cat} E$.

Theorem 1. Let $F \rightarrow E \stackrel{f}{\rightarrow} B$ as before. We suppose that cat $f<\infty$. Then

(1) there exists a nontrivial morphism of $H_{*}(\Omega B ; k)$-modules

$$
H_{*}(F ; k) \rightarrow H_{*}(\Omega B ; k),
$$

(2) there exists a subalgebra $K$ of $H_{*}(\Omega B ; k)$ such that $H_{*}(F ; k)$ contains a free $K$-module and such that $H_{*}(\Omega B ; k)$ is a finite-dimensional free $K$-module.

Theorem 2. Let $F \rightarrow E \rightarrow B$ as before. Suppose that $E$ has the homotopy type of a finite $C W$ complex and $\Omega B$ is a product of Eilenberg-Mac Lane spaces. Then the algebra $H_{*}(\Omega B ; k)$ is isomorphic to $K \otimes G$ with $G$ finite-dimensional and $H_{*}(F ; K)$ is a free $K$-module.

One can remark that if $f$ is homotopically trivial, then $F$ has the homotopy type of the product $\Omega B \times E$, and Theorem 1 is obviously true in this case. The point is that the homology of the fibre $F$ is never very far from being free; this is the content of the results.

In [3] Halperin and the authors establish a relation between cat $f$ and some homological invariants of $H_{*}(F ; k)$ as a module over $H_{*}(\Omega B ; k)$. Let $G=$ $\bigoplus_{i \geq 0} G_{i}$ be a graded Hopf algebra over the field $k$ satisfying

$$
\begin{aligned}
& G_{0}=k ; \\
& \operatorname{dim} G_{i}<\infty \text { for any } i \\
& G \text { is cocommutative. }
\end{aligned}
$$

The grade of a graded $G$-module $M$ is the greatest $n$ (or $\infty$ ) such that $\operatorname{Ext}_{G}^{n}(M ; G) \neq 0$. The depth of the Hopf algebra $G$ is, by definition, the grade of the trivial module $k$. The main result of [3] reads as follows.

Theorem [3, Theorem A]. With the above hypothesis, grade $\left(H_{*}(F ; k)\right) \leq$ cat $f$.

Following Moore and Smith [7], we call a Hopf algebra $G$ p-solvable if there exists a sequence of normal sub-Hopf algebras

$$
k \subset G_{\langle-s\rangle} \subset G_{\langle-s+1\rangle} \subset \cdots \subset G_{\langle 0\rangle}=G
$$

such that each quotient $G_{\langle t\rangle} / / G_{\langle t-1\rangle}$ is a commutative Hopf algebra with $x^{p}=$ 0 for every $x$ in $G_{\langle t\rangle} / / G_{\langle t-1\rangle}$, i.e., each quotient is a coprimitive Hopf algebra. The interest of $p$-solvable Hopf algebras in topology comes from the following result of Moore and Smith.

Theorem [7, Theorem 6.2]. If $\Omega X$ is a stable r-stage Postnikov system, then the Hopf algebra $H_{*}(\Omega X ; k)$ is p-solvable.

Every finitely generated coprimitive Hopf algebra is finite dimensional. Thus from Lemma 1, every finitely generated $p$-solvable Hopf algebra is also finite dimensional. In particular, each element has finite height.

Lemma 1. Let $G$ be a finitely generated Hopf algebra and $K$ be a normal subHopf algebra such that the quotient $G / / K$ is finite dimensional. Then $K$ is also finitely generated. 
Proof. From the Hoschchild-Serre spectral sequence associated to the short exact sequence of Hopf algebras

$$
K \rightarrow G \rightarrow G / / K
$$

we obtain an isomorphism

$$
\operatorname{Tor}_{G}^{1}(k, k) \cong \operatorname{Tor}_{G / / K}^{1}(k, k) \oplus\left(\operatorname{Tor}_{G / / K}^{0}\left(k, \operatorname{Tor}_{K}^{1}(k, k)\right) / \operatorname{Im}\left(d_{2}\right)\right) .
$$

As $G / / K$ is finite dimensional, the dimension of the vector space $\operatorname{Tor}_{G / / K}^{i}(k, k)$ is finite for every $i$; therefore, $\operatorname{Tor}_{G}^{1}(k, k)$ is finite dimensional if and only if $\operatorname{Tor}_{K}^{1}(k, k)$ is finite dimensional.

The next lemma is the main tool in the proof of Theorem 1. This is a generalization of [2, Proposition 3.1] with exactly the same proof.

Lemma 2. Suppose $0 \neq \omega \in \operatorname{Ext}_{G}^{m}(M, G), G$ a Hopf algebra, and $M$ a $G$ module. Then for some finitely generated sub-Hopf algebra $K \subset G, \omega$ restricts to a nonzero element of $\operatorname{Ext}_{K}^{m}(M, G)$.

Lemma 3. $A$ module $M$ of finite grade on a p-solvable Hopf algebra $G$ has grade zero.

Proof. We denote by $\omega$ a nonzero element in $\operatorname{Ext}_{G}^{m}(M, G)$. It then results from Lemma 2 that, for some finitely generated sub-Hopf algebra $H \subset G, \omega$ restricts to a nonzero element in $\operatorname{Ext}_{H}^{m}(M, G)$; therefore, $\operatorname{Ext}_{H}^{m}(M, H) \neq 0$.

Write $M=\lim M^{\alpha}$ with each $M^{\alpha}$ a finitely generated $A$-module. From the canonical isomorphisms

$$
\operatorname{Ext}_{H}^{s}(M, H)=\underset{\lim }{\longleftarrow} \operatorname{Ext}_{H}^{s}\left(M^{\alpha}, H\right),
$$

one can see that if $\operatorname{Ext}_{H}^{m}(M, H) \neq 0$, then $\operatorname{Ext}_{H}^{m}\left(M^{\alpha}, H\right) \neq 0$ for some finitely generated submodule $M^{\alpha}$.

The Hopf algebra $H$ is $p$-solvable and finitely generated; therefore, $H$ is finite dimensional and, hence, elliptic in the sense of [5]. By [4, Lemma 3.10] $m=0$.

Proof of Theorem 1. As the category of $p$ is finite, the grade of the $H_{*}(\Omega B ; k)$ module $H_{*}(F ; k)$ is finite; therefore, by Lemma 3 the grade is zero. This implies the existence of a nontrivial morphism of $H_{*}(\Omega B ; k)$-module $g: H_{*}(F ; k)$ $\rightarrow H_{*}(\Omega B ; k)$.

For the sake of simplicity we denote $G=H_{*}(\Omega B ; k)$ and $M=H_{*}(F ; k)$. By hypothesis there exists a sequence of normal sub-Hopf algebras

$$
k \subset A_{\langle-s\rangle} \subset A_{\langle-s+1\rangle} \subset \cdots \subset A_{\langle 0\rangle}=G
$$

such that each quotient $A_{\langle t\rangle} / / A_{\langle t-1\rangle}$ is isomorphic as an algebra to

$$
A_{\langle t\rangle} / / A_{\langle t-1\rangle} \cong \bigotimes_{i \in I_{t}} \Lambda x_{i} \otimes \bigotimes_{j \in J_{t}} k\left[y_{j}\right] / y_{j}^{p}
$$

For each degree $q$ we denote by $K_{q}$ the algebra generated by the $x_{i}$ and the $y_{j}$ of degree larger then $q$. By the previous decomposition, $G$ is a free finitely generated $K_{q}$-module for each integer $q$.

Let $m$ be an element of $M$ such that $g(m) \neq 0$. The element $g(m)$ belongs to a finitely generated subalgebra $H$ of $G$. Denote by $t$ the maximal degree of 
the homogeneous elements of $H$. As $G$ is a $K_{t}$ free module, $K_{t} \cdot g(m) \cong K_{t}$. This implies that the $K_{t}$-module generated by $m$ in $M$ is free.

Proof of Theorem 2. The homology Serre spectral sequence of the fibration

$$
\Omega B \rightarrow F \rightarrow E
$$

is a spectral sequence of $H_{*}(\Omega B ; k)$-modules. As $H_{*}(E ; k)$ is finite dimensional, there is only a finite number of nonzero differentials $d_{r}$ in this spectral sequence. We will prove by induction on $r$ that each $E_{r}$ is a free finitely generated module over some subalgebra $H_{r}$ of $G=H_{*}(\Omega B ; k)$ such that $G$ is a free finitely generated $H_{r}$-module.

This is true for $E_{2}$. The algebra $H_{*}(\Omega B ; k)$ is a tensor product $\bigotimes_{i} \Lambda x_{i} \otimes$ $\bigotimes_{j} k\left[y_{j}\right] / y_{j}^{p}$. The integer $q$ will be defined by

$q=\left(\right.$ the maximal degree of the homogeneous elements of $\left.H_{*}(E ; k)\right)+2$.

Denote by $\mathrm{H}_{3}$ the tensor product of the components $\Lambda x_{i}$ and $k\left[y_{j}\right] / y_{j}^{p}$ with $x_{i}$ and $y_{j}$ of degree greater than $q . G$ is then the tensor product $G=H_{3} \otimes$ $R_{3}$ with $R_{3}$ finite-dimensional. The $E_{2}$-term of the Serre spectral sequence, $\left(E_{2}, d_{2}\right)$, is then isomorphic to $\left(H_{3}, 0\right) \otimes\left(H_{*}(E ; k) \otimes R_{3}, d_{2}\right)$ as an $H_{3}$-module. Its homology $E_{3}$ is therefore isomorphic to $H_{3} \otimes H\left(H_{*}(E ; k) \otimes R_{3}, d_{2}\right)$ and is a free finitely generated $H_{3}$-module.

We proceed in exactly the same way for the general case. At each stage $H_{r}$ will be the intersection of $H_{r-1}$ and the tensor product of the components $\Lambda x_{i}$ and $k\left[y_{j}\right] / y_{j}^{p}$ with $x_{i}$ and $y_{j}$ of degree greater than $2+$ (the maximal degree of the generators of $E_{r-1}$ as an $H_{r-1}$-module).

\section{REFERENCES}

1. I. Bernstein and T. Ganea, The category of a map and of a cohomology class, Fund. Math. 50 (1962), 265-279.

2. Y. Félix, S. Halperin, and J. C. Thomas, Hopf algebras of polynomial growth, J. Algebra 125 (1989), 408-417.

3. _ - The category of a map and the grade of a module, Israel J. Math. (to appear).

4. _ Lie algebras of polynomial growth, J. London Math. Soc. 43 (1991), 556-566.

5. __ Elliptic Hopf algebras, J. London Math. Soc. 43 (1991), 545-555.

6. J. Milnor and J. C. Moore, On the structure of Hopf algebras, Ann. of Math. (2) 81 (1965), 211-264.

7. J. C. Moore and L. Smith, Hopf algebras and multiplicative fibrations. I, Amer. J. Math. 90 (1968), 752-780.

8. G. Whitehead, Elements of homotopy theory, Springer-Verlag, Berlin, Heidelberg, and New York, 1978.

Departmente Mathématique, Université Catholique de louvain, Belgium

Departmente Mathématique, Université des Sciences et Techniques de Lille, France 